\title{
Reply: Breast-feeding and allergy
}

\section{Bengt Björkstén and Bo Lundbäck}

Lung and Allergy Research, Institute of Environmental Medicine, Karolinska Institutet, Stockholm, Sweden

We would once again like to comment the paper by Kull et al. (1), in which the authors suggest that breast-feeding decreases the risk for development of eczema and discuss in general terms that breastfeeding protects against the development of allergy. Our criticism was based on the fact that exclusive breast-feeding for 4 months or more did not reduce the risk for eczema at the age of 4 years [odds ratio (OR) 0.88 , not significant) [2]. In order to avoid all possible disease-related modification of exposure, the authors excluded all children who developed eczema or asthma during the period of breastfeeding. By excluding those children who developed symptoms while they were breast-fed and by creating an arbitrary group with "severe allergic disease", the authors found support for a protective role of breast-feeding yielding a slightly lower OR of 0.78 .

The authors have now replied to our comments (3) by referring to two meta-analyses, one about breastfeeding and asthma (4) and another about breastfeeding and eczema (5). It is well known from several studies that breast-feeding protects against wheezing, at least during infancy. However, the problem is that recurrent wheezing has different phenotypes. The evidence quoted by Kull et al. refers to the non-allergic phenotype. This has also been shown in studies in Sweden by Rönmark et al. (6).

In her reply (3) to our initial critical remarks, Kull exemplifies the previous results by pooling asthma and eczema together and makes a point of the fact that there is no statistically significant difference between the association of breast-feeding and eczema whether children with an overlap and exposure are excluded or not. Such secondary analyses are open to critique, particularly in the absence of any power calculations. Non-allergic asthma is particularly common in very small children. By including non-allergic asthmatics, the results become biased when discussing protection against allergy. The fact is that there is little or no support for any major impact of breast-feeding on allergy development. The study by Kull et al. (1) has not contributed novel data on that point.
There is a problem with the meta-analysis related to eczema (5) quoted by Kull in that five of the papers providing low odds ratios for childhood eczema to be associated with breast-feeding were conducted by Chandra, whose work has been revealed to be fraudulent (7). Obviously Kull could not possibly have known this at the time of writing her paper, but the fact nevertheless provides further reason for our argument that there is little support for any significant allergy-preventive effect of breast-feeding. We would like to reaffirm our initial statement that preventive measures and information should be based on solid scientific ground, just as other medical therapies and interventions.

\section{References}

1. Kull I, Bohme M, Wahlgren CF, Nordvall L, Pershagen G, Wickman M. Breast-feeding reduces the risk for childhood eczema. J Allergy Clin Immunol 2005; 116 : 657-61.

2. Björksten B, Lundbäck B. Promoting breast-feeding with weak arguments. Scand J Food Nutr 2006;50:48.

3. Kull I. Reply: Breast-feeding and childhood eczema. Scand J Food Nutr 2006;50:49-50.

4. Gdalevich M, Mimouni D, Mimouni M. Breast-feeding and the risk of bronchial asthma in childhood: a systemic review and meta-analysis of prospective studies. J Pediatr 2001; 139: 261-6.

5. Gdalevich M, Mimouni D, David M, Mimouni M. Breast-feeding and the onset of atopic dermatitis in childhood: a systemic review and meta-analysis of prospective studies. J Allergy Clin Immunol 2005; 116: 657-61.

6. Rönmark E, Jönsson E, Platts-Mills TAE, Lundbäck B. Different risk factor pattern in atopic and non-atopic children with asthma - report from the Obstructive Lung Disease in Northern Sweden Study. Allergy 1999;9:92635.

7. http://www.cbc.ca/national/news/chandra/ 